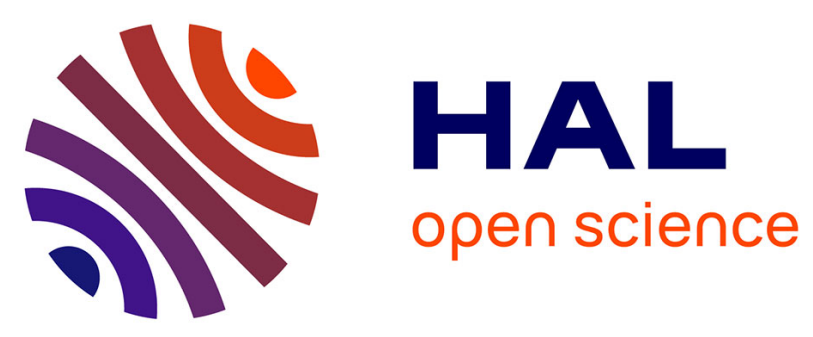

\title{
Spectral-Energy Efficiency Pareto Front in Cellular Networks: A Stochastic Geometry Framework
} Marco Di Renzo, Alessio Zappone, Thanh Tu Lam, Merouane Debbah

\section{To cite this version:}

Marco Di Renzo, Alessio Zappone, Thanh Tu Lam, Merouane Debbah. Spectral-Energy Efficiency Pareto Front in Cellular Networks: A Stochastic Geometry Framework. IEEE Wireless Communications Letters, 2019, 8 (2), pp.424-427. 10.1109/LWC.2018.2874642 . hal-02395683

\section{HAL Id: hal-02395683 https://hal.science/hal-02395683}

Submitted on 6 Jul 2020

HAL is a multi-disciplinary open access archive for the deposit and dissemination of scientific research documents, whether they are published or not. The documents may come from teaching and research institutions in France or abroad, or from public or private research centers.
L'archive ouverte pluridisciplinaire HAL, est destinée au dépôt et à la diffusion de documents scientifiques de niveau recherche, publiés ou non, émanant des établissements d'enseignement et de recherche français ou étrangers, des laboratoires publics ou privés. 


\title{
Spectral-Energy Efficiency Pareto Front in Cellular Networks: A Stochastic Geometry Framework
}

\author{
Marco Di Renzo, Senior Member, IEEE, Alessio Zappone, Senior Member, IEEE, \\ Thanh Tu Lam, Student Member, IEEE, and Mérouane Debbah, Fellow, IEEE
}

\begin{abstract}
We compute the spectral efficiency vs. energy efficiency Pareto front in Poisson cellular networks, by formulating a spectral-energy efficiency bi-objective optimization problem as a function of either the transmit power or the density of the base stations. Capitalizing on fundamental results on weighted Tchebycheff optimization problems applied to strictly quasiconcave functions, we derive analytical expressions of the unique Pareto-optimal solution of the bi-objective problem. We prove that the Pareto front is constituted by a subset of the spectralenergy efficiency trade-off curve and that it can be formulated in analytical terms. We identify new functional relations between the Pareto-optimal transmit power and density of the base stations. Index Terms-Cellular network, Pareto front, point process.
\end{abstract}

\section{INTRODUCTION}

The Spectral Efficiency (SE) and Energy Efficiency (EE) are important performance metrics that guide the optimization of cellular networks. Under typical operating conditions, however, they are conflicting objective functions [1]: There exists no single solution that simultaneously optimizes each of them. There exist, on the other hand, several optimal solutions for which none of the two objective functions can be improved without degrading the other objective. The SEEE pairs that fulfill the latter optimality condition are referred to as Pareto-optimal solutions, and the corresponding SE-EE curve is known as the Pareto front [2, Def. 2.2.1]. Since all Pareto-optimal solutions are, without any subjective preference information, equally good, it is important to identify the entire Pareto front for subsequent (subjective) decision making.

The aim of this letter is to derive a complete and explicit formulation of the SE-EE Pareto front in cellular networks. We focus our attention on analytically formulating the SEEE Pareto front from the system-level standpoint, i.e., by taking the average with respect to the irregular deployments of cellular Base Stations (BSs) and the random locations of the Mobile Terminals (MTs) within the cells. Some authors have recently studied the SE-EE trade-off in cellular networks from the system-level standpoint [3]-[5]. The contribution of this letter is, however, different: We are not interested in analyzing the SE-EE trade-off but in characterizing the SE-EE Pareto front, which is the solution of a bi-objective optimization problem [2]. Recently, the authors of [6] and [7] have computed the SE-EE Pareto front in cellular networks with the aid of multi-objective optimization theory. Therein, however, numerical methods are used, and, thus, no explicit analytical formulation of the SE-EE Pareto front is given.

Manuscript received June 8, 2018. This work was supported in part by the European Commission through the H2020-MSCA ETN-5Gwireless project under grant 641985, the H2020-MSCA IF-BESMART project under grant 749336, and the H2020-ERC PoC-CacheMire project under grant 727682.

The authors are with the Laboratoire des Signaux et Systèmes, CNRS CentraleSupélec, Univ Paris Sud, Université Paris-Saclay, 3 rue Joliot-Curie, 91192 Gif-sur-Yvette, France. (e-mail: marco.direnzo@12s.centralesupelec.fr).
TABLE I

Notation ( $\alpha=3.5, \delta=2 / \beta, \beta>2, \eta=\kappa \sigma_{\mathrm{N}}^{2} \gamma_{\mathrm{A}}, \dot{f}:$ 1St DeRIVATIVE).

\begin{tabular}{|l||l|}
\hline \multicolumn{1}{|c||}{ Symbol/Function } & \multicolumn{1}{c|}{ Definition } \\
\hline \hline$\lambda_{\mathrm{BS}}, \lambda_{\mathrm{MT}}$ & Density of BSs, density of MTs \\
\hline $\mathrm{P}_{\mathrm{tx}}, \mathrm{B}_{\mathrm{W}}$ & Transmit power, transmission bandwidth \\
\hline $\mathrm{N}_{0}, \sigma_{\mathrm{N}}^{2}=\mathrm{B}_{\mathrm{W}} \mathrm{N}_{0}$ & Noise power spectral density, noise variance \\
\hline $\mathrm{P}_{\mathrm{c}}, \mathrm{P}_{\mathrm{i}}\left(\mathrm{P}_{\mathrm{c}} \geq \mathrm{P}_{\mathrm{i}}\right)$ & Circuits and idle power consumption of BSs \\
\hline$\kappa, \beta$ & Path-loss constant and slope \\
\hline$\gamma_{\mathrm{D}}, \gamma_{\mathrm{A}}$ & Threshold for decoding and cell association \\
\hline${ }_{2} F_{1}(\cdot, \cdot, \cdot, \cdot)$ & Gauss hypergeometric function \\
\hline$\rho_{\mathrm{D}}, \mathcal{L}(x)$ & $\log 2\left(1+\gamma_{\mathrm{D}}\right), 1-(1+x / \alpha)-\alpha$ \\
\hline$\Upsilon$ & $2 F_{1}\left(-\delta, 1,1-\delta,-\gamma_{\mathrm{D}}\right)-1 \geq 0$ \\
\hline $\mathcal{Q}(x, y, z)$ & $1-\exp \left(-\pi x(y / \eta)^{\delta}(1+\Upsilon \mathcal{L}(z))\right)$ \\
\hline $\mathrm{P}_{\mathrm{grid}}$ & $\lambda_{\mathrm{BS}}\left(\mathrm{P}_{\mathrm{tx}}-\mathrm{P}_{\mathrm{i}}\right) \mathcal{L}\left(\lambda_{\mathrm{MT}} / \lambda_{\mathrm{BS}}\right)+\lambda_{\mathrm{MT}} \mathrm{P}_{\mathrm{c}}+\lambda_{\mathrm{BS}} \mathrm{P}_{\mathrm{i}}$ \\
\hline $\mathrm{SE}_{\mathrm{P}}^{(\infty)}$ & $\mathrm{B}_{\mathrm{W}} \rho_{\mathrm{D}} \lambda_{\mathrm{BS}} \mathcal{L}\left(\lambda_{\mathrm{MT}} / \lambda_{\mathrm{BS}}\right) /\left(1+\Upsilon \mathcal{L}\left(\lambda_{\mathrm{MT}} / \lambda_{\mathrm{BS}}\right)\right)$ \\
\hline $\mathrm{SE}_{\lambda_{\mathrm{BS}}^{(\infty)}}^{(m)}, \mathrm{B}_{\mathrm{W}} \rho_{\mathrm{D}} \lambda_{\mathrm{MT}}$ \\
\hline $\mathrm{P}_{\mathrm{tx}}^{(\min )}, \mathrm{P}_{\mathrm{tx}}^{(\max )}$ & Minimum and maximum values of $\mathrm{P}_{\mathrm{tx}}$ \\
\hline$\lambda_{\mathrm{BS}}^{(\min )}, \lambda_{\mathrm{BS}}^{(\max )}$ & Minimum and maximum values of $\lambda_{\mathrm{BS}}$ \\
\hline
\end{tabular}

Against this background, we derive an explicit analytical formulation of the SE-EE Pareto front in cellular networks, which is obtained by solving a SE-EE bi-objective optimization problem, as a function of either the transmit power or the deployment density of the BSs, without resorting to any numerical methods. To the best of the authors' knowledge, this has been a long-lasting open research issue. Our new contribution is obtained by capitalizing on the approach recently introduced in [8] for computing, in closed-form, the SE and EE in Poisson cellular networks, and on fundamental theoretical results on the existence and uniqueness of Pareto-optimal solutions in weighted Tchebycheff optimization problems [2].

\section{Bi-Objective Problem Formulation}

We consider a cellular network whose BSs and MTs are distributed according to two mutually independent homogeneous Poisson Point Processes (PPPs) of density $\lambda_{\mathrm{BS}}$ and $\lambda_{\mathrm{MT}}$, respectively. The same system model as in [8] is assumed. By using the notation in Table I, the SE $\left(\mathrm{bit} / \mathrm{sec} / \mathrm{m}^{2}\right)$ and the $\mathrm{EE}$ (bit/Joule) can be formulated, respectively, as follows:

$$
\begin{array}{r}
\mathrm{SE}=\mathrm{B}_{\mathrm{W}} \rho_{\mathrm{D}} \frac{\lambda_{\mathrm{BS}} \mathcal{L}\left(\lambda_{\mathrm{MT}} / \lambda_{\mathrm{BS}}\right)}{1+\Upsilon \mathcal{L}\left(\lambda_{\mathrm{MT}} / \lambda_{\mathrm{BS}}\right)} \mathcal{Q}\left(\lambda_{\mathrm{BS}}, \mathrm{P}_{\mathrm{tx}}, \frac{\lambda_{\mathrm{MT}}}{\lambda_{\mathrm{BS}}}\right) \\
\mathrm{EE}=\frac{\mathrm{SE}}{\lambda_{\mathrm{BS}}\left(\mathrm{P}_{\mathrm{tx}}-\mathrm{P}_{\mathrm{i}}\right) \mathcal{L}\left(\lambda_{\mathrm{MT}} / \lambda_{\mathrm{BS}}\right)+\lambda_{\mathrm{MT}} \mathrm{P}_{\mathrm{c}}+\lambda_{\mathrm{BS}} \mathrm{P}_{\mathrm{i}}}
\end{array}
$$

where the denominator in (2), i.e., $\mathrm{P}_{\text {grid }}$, is the power consumption $\left(\mathrm{Watt} / \mathrm{m}^{2}\right)$ of the cellular network [8].

In Table II, we summarize important properties of the SE and $\mathrm{EE}$, as a function of $\mathrm{P}_{\mathrm{tx}}$ and $\lambda_{\mathrm{BS}}$, that are used next. For generality, we use the symbol $\xi$ to denote either $\mathrm{P}_{\mathrm{tx}}$ or $\lambda_{\mathrm{BS}}$. In particular, the unique maximizer of $\mathrm{EE}, \xi^{(\circ)}$, is [8]:

$$
\xi^{(\mathrm{o})}=\xi^{(\mathrm{EE}, \mathrm{opt})}=\max \left\{\xi^{(\mathrm{min})}, \min \left\{\xi^{(*)}, \xi^{(\max )}\right\}\right\}
$$


TABLE II

MAIN PROPERTIES OF SE \& EE $\left(\xi=\mathrm{P}_{\mathrm{tx}}\right.$ OR $\xi=\lambda_{\mathrm{BS}}, \xi^{(\min )} \& \xi^{(\max )}$ ARE THE MINIMUM \& MAXIMUM VALUES OF $\xi, \Omega=\left[\xi^{(\min )}, \xi^{(\max )}\right]$, $\left.\Omega^{(\min )}=\left[\xi^{(\min )}, \xi^{(\mathrm{o})}\right], \Omega^{(\max )}=\left[\xi^{(\mathrm{o})}, \xi^{(\max )}\right]\right)$.

\begin{tabular}{|c|c|}
\hline Property & Meaning \\
\hline$\overline{\mathrm{SE}}(\xi=0)=0$ & SE is zero if $\xi=0$ \\
\hline $\mathrm{SE}\left(\mathrm{P}_{\mathrm{tx}} \rightarrow \infty\right)=\mathrm{SE}_{\mathrm{P}_{\mathrm{tx}}}^{(\infty)}$ & Asymptotic limit of SE with $\mathrm{P}_{\mathrm{tx}}$ \\
\hline $\mathrm{SE}\left(\lambda_{\mathrm{BS}} \rightarrow \infty\right)=\mathrm{SE}_{\lambda_{\mathrm{BS}}}^{(\infty)}$ & Asymptotic limit of SE with $\lambda_{\mathrm{BS}}$ \\
\hline$\dot{\mathrm{SE}}(\xi>0)>0$ & SE is strictly increasing if $\xi>0$ \\
\hline$\xi^{(\mathrm{m})}=\xi^{(\mathrm{SE}, \mathrm{opt})}=\xi^{(\max )}$ & Unique maximizer of $\mathrm{SE}$ in $\xi \in \Omega$ \\
\hline $\mathrm{SE}_{\mathrm{m}}=\mathrm{SE}\left(\xi^{(\mathrm{m})}\right)$ & Maximum value of $\mathrm{SE}$ in $\xi \in \Omega$ \\
\hline $\mathrm{EE}(\xi=0)=0$ & EE is zero if $\xi=0$ \\
\hline $\mathrm{EE}(\xi \rightarrow \infty)=0$ & EE is zero if $\xi \rightarrow \infty$ \\
\hline $\mathrm{EE}(\xi)$ is unimodal in $\xi$ & EE has a unique maximizer (see (3)) \\
\hline$\xi^{(\mathrm{o})}=\xi^{(\mathrm{EE}, \mathrm{opt})}=(3)$ & Unique maximizer of EE for $\xi \in \Omega$ \\
\hline $\operatorname{EE}(\xi)>0, \xi \in \Omega^{(\min )}$ & $\mathrm{EE}$ is strictly increasing if $\xi \in \Omega^{(\mathrm{min})}$ \\
\hline $\mathrm{EE}(\xi)<0, \xi \in \Omega^{(\max )}$ & EE is strictly decreasing if $\xi \in \Omega^{(\max )}$ \\
\hline $\mathrm{EE}_{\mathrm{o}}=\mathrm{EE}\left(\xi^{(\mathrm{o})}\right)$ & Maximum value of EE in $\xi \in \Omega$ \\
\hline
\end{tabular}

where $\xi^{(*)}$ is the unique unconstrained maximizer of the EE. The trends in Table II either are proved in [8] or directly follow from (1) and (2). In this letter, thus, no proof is given. We note that the SE and $\mathrm{EE}$ are continuous functions in $\xi \in \Omega$.

We are interested in solving, as a function of $\xi=\mathrm{P}_{\mathrm{tx}}$ or $\xi=\lambda_{\mathrm{BS}}$, the following bi-objective optimization problem [2]:

$$
\mathcal{P}: \max _{\xi \in \Omega}[\mathrm{SE}(\xi), \mathrm{EE}(\xi)]
$$

Definition 1: The bi-objective optimization problem in (4) is said to be non-trivial or meaningful if $\xi^{(\mathrm{o})}<\xi^{(\max )}$.

Remark 1: If $\xi^{(\mathrm{o})} \geq \xi^{(\max )}, \mathcal{P}$ in (4) is trivial because, based on Table II, both the SE and EE are increasing functions in $\xi$. Thus, they are not conflicting objectives and are both maximized for $\xi=\xi^{(\max )}$, i.e., the (trivial) solution of (4). $\square$

Remark 2: Strictly monotonically increasing and unimodal functions are strictly quasi-concave functions [9].

From Remark 2, we evince that the SE and EE are strictly quasi-concave functions. This property is used in the sequel. In the next sections, we focus our attention on non-trivial biobjective optimization problems. For generality, where appropriate, we use the mapping $f(\xi)=\mathrm{SE}(\xi)$ and $g(\xi)=\mathrm{EE}(\xi)$.

\section{TCHEBYCHEFF SCALARIZATION}

To solve $\mathcal{P}$ and compute the Pareto-front, we employ the scalarization approach [2]. Two methods are used: 1) the conventional weighted Tchebycheff optimization $\left(\mathcal{P}_{\mathrm{T}}\right)[2, \mathrm{Sec}$. 3.4], and 2) the simplified weighted Tchebycheff optimization $\left(\mathcal{P}_{\mathrm{ST}}\right) . \mathcal{P}_{\mathrm{ST}}$ is introduced in this letter and proved to be equivalent to $\mathcal{P}_{\mathrm{T}}$, but shown to be instrumental for obtaining an analytical expression of the Pareto front. $\mathcal{P}_{\mathrm{T}}$ is studied first in order to mathematically prove the equivalence with $\mathcal{P}_{\mathrm{ST}}$.

Lemma 1: Let $f(\xi)$ and $g(\xi)$ be two strictly quasi-concave functions in $\xi \in \Omega$. Then, the point-wise minimum function, $\mathcal{X}(\xi)=\min \{f(\xi), g(\xi)\}$, is strictly quasi-concave in $\xi \in \Omega$.

Proof: Let us define $\xi_{a}=a \xi_{1}+(1-a) \xi_{2}, \mathcal{X}_{f}\left(\xi_{1}, \xi_{2}\right)=$ $\min \left\{f\left(\xi_{1}\right), f\left(\xi_{2}\right)\right\}, \mathcal{X}_{g}\left(\xi_{1}, \xi_{2}\right)=\min \left\{g\left(\xi_{1}\right), g\left(\xi_{2}\right)\right\}$. Вy virtue of strict quasi-concavity, $f\left(\xi_{a}\right)>\mathcal{X}_{f}\left(\xi_{1}, \xi_{2}\right), g\left(\xi_{a}\right)>$ $\mathcal{X}_{g}\left(\xi_{1}, \xi_{2}\right)$ for $\xi_{1} \neq \xi_{2} \in \Omega$ and $a \in(0,1)$. Then, $\mathcal{X}\left(\xi_{a}\right)=$ $\min \left\{f\left(\xi_{a}\right), g\left(\xi_{a}\right)\right\}>\min \left\{\mathcal{X}_{f}\left(\xi_{1}, \xi_{2}\right), \mathcal{X}_{g}\left(\xi_{1}, \xi_{2}\right)\right\}=$ $\min \left\{\mathcal{X}\left(\xi_{1}\right), \mathcal{X}\left(\xi_{2}\right)\right\}$, where the inequality holds true because the point-wise minimum is an increasing function, and $f(\cdot)$ and $g(\cdot)$ are strictly quasi-concave functions. The last equality is obtained by definition of point-wise minimum.

Lemma 2: Let $f(\xi)$ be a strictly quasi-concave function in $\xi \in \Omega$. Then, $f(\xi)$ has a unique maximizer in $\xi \in \Omega$.

Proof: It follows from [9, Corollary 2.5.1].

Proposition 1: Let $f(\xi)$ and $g(\xi)$ be two strictly quasiconcave functions in $\xi \in \Omega$. Then, the optimization problem:

$$
\mathcal{P}_{\max -\min }: \max _{\xi \in \Omega}\{\min \{f(\xi), g(\xi)\}\}
$$

has a unique solution in $\xi \in \Omega$.

Proof: It follows from Lemma 1 and Lemma 2.

Let $f(\xi)$ be a strictly quasi-concave function in $\xi \in \Omega$. Similar to the notation in Table II, the unique maximizer of $f(\cdot)$ and its corresponding maximum objective value are denoted by $\xi^{(f, \mathrm{opt})}$ and $f_{\text {opt }}=f\left(\xi^{(f, \mathrm{opt})}\right)$, respectively.

\section{A. Weighted Tchebycheff Method}

Let $f(\xi)$ and $g(\xi)$ be two continuous functions in $\xi \in \Omega$. The (conventional) weighted Tchebycheff optimization problem is defined as follows [2, Section 3.4]:

$$
\mathcal{P}_{\mathrm{T}}: \max _{\xi \in \Omega}\{\min \{\mu F(\xi),(1-\mu) G(\xi)\}\}
$$

where $F(\xi)=f(\xi) / f_{\text {opt }}-1, G(\xi)=g(\xi) / g_{\text {opt }}-1$, and $\mu \in[0,1]$ is a given and constant parameter.

Lemma 3: Let $f(\xi)$ and $g(\xi)$ be two strictly quasi-concave functions in $\xi \in \Omega$. Then, $\mathcal{P}_{\mathrm{T}}$ in (6) has, for each $\mu \in[0,1]$, a unique solution that is (strong) Pareto-optimal.

Proof: It follows from Prop. 1 and [9, Cor. 3.4.4].

Proposition 2: Let $\xi^{(\mu)}$ be the unique solution of $\mathcal{P}_{\mathrm{T}}$ in (6) for $\mu \in[0,1]$. Let us consider $f(\xi)=\mathrm{SE}(\xi)$ and $g(\xi)=$ $\mathrm{EE}(\xi)$. The Pareto front of $\mathcal{P}$ in (4) is constituted by the pairs $\left(f\left(\xi^{(\mu)}\right), g\left(\xi^{(\mu)}\right)\right)$ that are obtained by varying $\mu \in[0,1]$.

Proof: It follows from [9, Theorem 3.4.5].

Lemma 4: Let $\xi^{(\mu)}$ be the unique solution of $\mathcal{P}_{\mathrm{T}}$ in (6) for $\mu \in[0,1]$. Let us consider $f(\xi)=\mathrm{SE}(\xi)$ and $g(\xi)=\mathrm{EE}(\xi)$. Then, $\xi^{(\mu)} \in\left[\xi^{(\mathrm{o})}, \xi^{(\max )}\right]$, where $\xi^{(\mathrm{o})}$ is given in (3).

Proof: In the range $\xi^{(\mu)}<\xi^{(\mathrm{o})}$, the SE and EE are increasing functions. Based on the definition of Pareto-optimality, the pairs $\left(f\left(\xi^{(\mu)}\right), g\left(\xi^{(\mu)}\right)\right)$ cannot be Pareto-optimal.

Lemma 5: Let $\xi^{(\mu)} \in\left[\xi^{(\mathrm{o})}, \xi^{(\max )}\right]$ be the unique solution of $\mathcal{P}_{\mathrm{T}}$ in (6) for $\mu \in[0,1]$. Let us consider $f(\xi)=\mathrm{SE}(\xi)$ and $g(\xi)=\mathrm{EE}(\xi)$. Then, we have: i) $\xi^{(\mu)}=\xi^{(g, \mathrm{opt})}=\xi^{(\mathrm{o})}$ if and only if $\mu=0$, ii) $\xi^{(\mu)}=\xi^{(f, \mathrm{opt})}=\xi^{(\mathrm{m})}$ if and only if $\mu=1$, and iii) $\xi^{(\mu)}$ is the unique solution of the equation $\mu F\left(\xi^{(\mu)}\right)=(1-\mu) G\left(\xi^{(\mu)}\right)$ if and only if $\mu \in(0,1)$.

Proof: If $\mu=0$ and $\mu=1, \mathcal{P}_{\mathrm{T}}$ in (6) is equivalent to maximizing $G(\cdot)$ and $F(\cdot)$, respectively, since, by definition, they are both negative functions. If $\mu \in(0,1)$, the SE is monotonically increasing and the $\mathrm{EE}$ is monotonically decreasing in the range $\left[\xi^{(\mathrm{o})}, \xi^{(\max )}\right]$. Also, $\mu F\left(\xi^{(\mathrm{o})}\right)<(1-\mu) G\left(\xi^{(\mathrm{o})}\right)=0$ and $(1-\mu) G\left(\xi^{(\max )}\right)<\mu F\left(\xi^{(\max )}\right)=0$. So, the functions $\mu F(\xi)$ and $(1-\mu) G(\xi)$ cross each other exactly once in $\left[\xi^{(\mathrm{o})}, \xi^{(\max )}\right]$. By definition of max-min optimization, this unique crossing point is the solution of $\mathcal{P}_{\mathrm{T}}$ in (6).

Lemma 6: Let $f(\xi)$ and $g(\xi)$ be two continuous and strictly quasi-concave functions in $\xi \in \Omega$. Then, there exists a continuous and strictly decreasing function, $\mathcal{C}$, that expresses the objective function $g$ in terms of the objective function $f$, 
i.e., $g=\mathcal{C}(f)$, where $f\left(\xi^{(g, \text { opt })}\right) \leq f \leq f_{\text {opt }}=f\left(\xi^{(f, \text { opt })}\right)$ and $g\left(\xi^{(f, \text { opt })}\right) \leq g \leq g_{\text {opt }}=g\left(\xi^{(g, \text { opt })}\right)$.

Proof: It follows from [10, Theorem 2.1, Theorem 2.2], since $f(\cdot)$ and $g(\cdot)$ are continuous and strictly quasi-concave functions, and $\Omega$ is a non-empty, compact, and convex set. $\square$

Remark 3: Proposition 2, Lemma 5, and Lemma 6 provide us with fundamental properties of the SE-EE Pareto front. They, however, have the following limitations: i) Proposition 2 does not yield an explicit analytical formulation of the Pareto front, which is parameterized as a function of $\mu$, ii) if $\mu \in(0,1)$, it is not straightforward to obtain a closed-form expression of $\xi^{(\mu)}$ from Lemma 5, and iii) Lemma 6 asserts the existence of the curve $\mathcal{C}$ but does not provide an explicit formula for it. Also, no insight for system design is obtained from them. $\square$

As anticipated, these limitations are overcome in the next section with the aid of the proposed $\mathcal{P}_{\mathrm{ST}}$ method.

\section{B. Simplified Weighted Tchebycheff Method}

Unless otherwise stated, we assume $f(\xi)=\mathrm{SE}(\xi)$ and $g(\xi)=\mathrm{EE}(\xi)$ in $\xi \in \Omega$. We introduce the simplified weighted Tchebycheff problem as follows (for any $w \in(0,1)$ ):

$$
\mathcal{P}_{\mathrm{ST}}: \max _{\xi \in \Omega^{(\max )}}\{\min \{w \bar{F}(\xi),(1-w) \bar{G}(\xi)\}\}
$$

where $\bar{F}(\xi)=\mathrm{SE}(\xi) / \mathrm{SE}_{\mathrm{m}}$ and $\bar{G}(\xi)=\mathrm{EE}(\xi) / \mathrm{EE}_{\mathrm{o}}$.

Remark 4: The optimization problem in (7) is restricted over the set $\xi \in \Omega^{(\max )}$ by virtue of Lemma 4, i.e., only the values $\xi \geq \xi^{(0)}$ are admissible in order for the pairs ( $\left.\mathrm{SE}(\xi), \mathrm{EE}(\xi)\right)$ not to contradict the definition of Pareto-optimality.

Remark 5: $\mathcal{P}_{\mathrm{ST}}$ in (7) fulfills the conditions stated in Proposition 1: It has a unique solution in $\xi \in \Omega^{(\max )}$ for every $w \in(0,1)$. No conclusion, however, can be drawn about its Pareto-optimality, i.e., Lemma 3 is, in general, not true.

The following lemma and proposition provide us with sufficient conditions under which $\mathcal{P}_{\mathrm{T}}$ and $\mathcal{P}_{\mathrm{ST}}$ describe the same SE-EE Pareto front as a function of $\mu$ and $w$, respectively.

Lemma 7: Define $w_{l}=\left(1+\mathrm{EE}_{\mathrm{o}} / \mathrm{EE}\left(\xi^{(\mathrm{m})}\right)\right)^{-1} \in(0,1)$ and $w_{u}=\left(1+\mathrm{SE}\left(\xi^{(0)}\right) / \mathrm{SE}_{\mathrm{m}}\right)^{-1} \in(0,1)$. The unique solution, $\xi^{(w)}$, of $\mathcal{P}_{\mathrm{ST}}$ in (7) is: i) $\xi^{(w)}=\xi^{(\mathrm{m})}$ if and only if $w \leq$ $w_{l}$, ii) $\xi^{(w)}=\xi^{(\text {o) }}$ if and only if $w \geq w_{u}$, and iii) the unique solution of the equation $w \bar{F}\left(\xi^{(w)}\right)=(1-w) \bar{G}\left(\xi^{(w)}\right)$ in $\xi^{(w)} \in \Omega^{(\max )}$ if and only if $w \in\left(w_{l}, w_{u}\right)$.

Proof: In $\Omega^{(\max )}=\left[\xi^{(\mathrm{o})}, \xi^{(\max )}\right]$, the SE and EE are monotonically increasing and decreasing, respectively. Then, for a given $w \in(0,1)$ : i) $\min \{w \bar{F}(\xi),(1-w) \bar{G}(\xi)\}=\bar{F}(\xi)$ if $w \bar{F}\left(\xi^{(\max )}\right) \leq(1-w) \bar{G}\left(\xi^{(\max )}\right)$, i.e., the maximum of $w \bar{F}(\xi)$ in $\xi \in \Omega^{(\max )}$ is less than the minimum of $(1-w) \bar{G}(\xi)$ in $\xi \in \Omega^{(\max )}$, which yields $w \leq w_{l}$, ii) $\min \{w \bar{F}(\xi),(1-w) \bar{G}(\xi)\}=\bar{G}(\xi)$ if $(1-w) \bar{G}\left(\xi^{(\circ)}\right) \leq$ $w \bar{F}\left(\xi^{(\circ)}\right)$, i.e., the maximum of $(1-w) \bar{G}(\xi)$ in $\xi \in \Omega^{(\max )}$ is less than the minimum of $w \bar{F}(\xi)$ in $\xi \in \Omega^{(\max )}$, which yields $w \geq w_{u}$, and iii) the functions $w \bar{F}(\xi)$ and $(1-w) \bar{G}(\xi)$ always cross each other exactly once in $\Omega^{(\max )}$ if $w \bar{F}\left(\xi^{(\mathrm{o})}\right)<(1-w) \bar{G}\left(\xi^{(\mathrm{o})}\right)$ and $w \bar{F}\left(\xi^{(\max )}\right)>$ $(1-w) \bar{G}\left(\xi^{(\max )}\right)$, since the $\mathrm{SE}$ and $\mathrm{EE}$ are monotonically increasing and decreasing functions, respectively, in $\Omega^{(\max )}$. By definition of max-min optimization, this unique crossing point is the solution of $\mathcal{P}_{\mathrm{ST}}$ in (7). By definition, $\mathrm{EE}_{\mathrm{o}} / \mathrm{EE}\left(\xi^{(\mathrm{m})}\right)>1, \mathrm{SE}\left(\xi^{(\mathrm{o})}\right) / \mathrm{SE}_{\mathrm{m}}<1$. So, $w_{l}<w_{u}$.
Proposition 3: Let $\xi^{(w)}$ be the unique solution of $\mathcal{P}_{\mathrm{ST}}$ in (7) according to Lemma 7. The pairs $\left(f\left(\xi^{(w)}\right), g\left(\xi^{(w)}\right)\right)$ obtained by varying $w \in\left[w_{l}, w_{u}\right]$ describe the same SEEE Pareto front as $\mathcal{P}_{\mathrm{T}}$ in (6). Given $w,\left(f\left(\xi^{(w)}\right), g\left(\xi^{(w)}\right)\right)$ is obtained from $\mathcal{P}_{\mathrm{T}}$ in (6) by choosing $1 / \mu=1+$ $\left(1 / w-\phi_{+}^{(w)}\right)\left(1 / \phi_{-}^{(w)}\right)$, where $\phi_{ \pm}^{(w)}=1 \pm 1 / \bar{G}\left(\xi^{(w)}\right)$.

Proof: From Lemma 5 and Lemma 7, $\mathcal{P}_{\mathrm{T}}$ and $\mathcal{P}_{\mathrm{ST}}$ provide the same $\left(f\left(\xi^{(w)}\right), g\left(\xi^{(w)}\right)\right)=\left(f\left(\xi^{(\mu)}\right), g\left(\xi^{(\mu)}\right)\right)$ pairs if and only if the equations $\mu F\left(\xi^{(\mu)}\right)=(1-\mu) G\left(\xi^{(\mu)}\right)$ and $w \bar{F}\left(\xi^{(w)}\right)=(1-w) \bar{G}\left(\xi^{(w)}\right)$ are simultaneously satisfied. By imposing this condition, we obtain $1 / \mu=1+$ $\left(1 / w-\phi_{+}^{(w)}\right)\left(1 / \phi_{-}^{(w)}\right)$. By direct inspection of this latter formula, we evince that: i) $\mu=1$ if and only if $w=w_{l}$, ii) $\mu=0$ if and only if $w=w_{u}$, and iii) $\mu \in(0,1)$ if and only if $w \in\left(w_{l}, w_{u}\right)$, since $\mu$ in the obtained formula decreases monotonically as $w$ increases. This completes the proof.

Remark 6: From Proposition 3, we evince that the SE-EE Pareto front of $\mathcal{P}_{\mathrm{T}}$ in (6) can be obtained from $\mathcal{P}_{\mathrm{ST}}$ in (7). It is constituted, in particular, by: i) the point $\left(\mathrm{SE}_{\mathrm{m}}, \mathrm{EE}\left(\xi^{(\mathrm{m})}\right)\right)$ if $w=w_{l}$ (extreme right value), ii) the point $\left(\mathrm{SE}\left(\xi^{(\mathrm{o})}\right), \mathrm{EE}_{\mathrm{o}}\right)$ if $w=w_{u}$ (extreme left value), and iii) the continuous set of points obtained by varying $w$ in the range $w \in\left(w_{l}, w_{u}\right)$.

Compared with $\mathcal{P}_{\mathrm{T}}$ in (6), the advantage of $\mathcal{P}_{\mathrm{ST}}$ in (7) is that, with the exception of the extreme left and right points of the SE-EE Pareto front that are known, the others are the unique solution of $w \bar{F}\left(\xi^{(w)}\right)=(1-w) \bar{G}\left(\xi^{(w)}\right)$ for $w \in$ $\left(w_{l}, w_{u}\right)$ and $\xi^{(w)} \in \Omega^{(\max )}$. This is the fundamental result that allows us to compute an explicit analytical formulation of the SE-EE Pareto front, as proved in the next section.

\section{SE-EE PARETO Front}

The following sections provide an explicit formulation of the SE-EE Pareto front for $\xi=\mathrm{P}_{\mathrm{tx}}$ and $\xi=\lambda_{\mathrm{BS}}$. We prove, in particular, that the SE-EE Pareto front can be computed by knowing only $\xi^{(\mathrm{m})}=\xi^{(\max )}$ and $\xi^{(\mathrm{o})}$ available from [8].

\section{A. Case Study $\xi=\mathrm{P}_{\mathrm{tx}}$}

Lemma 8: Let $\xi=\mathrm{P}_{\mathrm{tx}}$ and $w \in\left[w_{l}, w_{u}\right]$, where $w_{l}$ and $w_{u}$ are defined in Lemma 7. Define $\mathrm{P}_{\mathrm{tx}}^{(\mathrm{o})}=\xi^{(o)}$. The unique solution, $\xi^{(w)}=\mathrm{P}_{\mathrm{tx}}^{(w)}$, of $\mathcal{P}_{\mathrm{ST}}$ in (7) is $\mathrm{P}_{\mathrm{tx}}^{(w)}=\mathrm{P}_{\mathrm{tx}}^{(\mathrm{o})}$ if $w=w_{u}$ and $\mathrm{P}_{\mathrm{tx}}^{(w)}=\mathrm{P}_{\mathrm{tx}}^{(\max )}$ if $w=w_{l}$. If $w \in\left(w_{l}, w_{u}\right)$, it is:

$$
\mathrm{P}_{\mathrm{tx}}^{(w)}=\frac{(1 / w-1)\left(\mathrm{SE}_{\mathrm{m}} / \mathrm{EE}_{\mathrm{o}}\right)-\mathcal{T}}{\lambda_{\mathrm{BS}} \mathcal{L}\left(\lambda_{\mathrm{MT}} / \lambda_{\mathrm{BS}}\right)} \in\left(\mathrm{P}_{\mathrm{tx}}^{(\mathrm{o})}, \mathrm{P}_{\mathrm{tx}}^{(\max )}\right)
$$

where $\mathcal{T}=\lambda_{\mathrm{MT}} \mathrm{P}_{\mathrm{c}}+\lambda_{\mathrm{BS}} \mathrm{P}_{\mathrm{i}}\left(1-\mathcal{L}\left(\lambda_{\mathrm{MT}} / \lambda_{\mathrm{BS}}\right)\right)$.

Proof: The cases $w=w_{l}$ and $w=w_{u}$ follow from Lemma 7. As for $w \in\left(w_{l}, w_{u}\right),(8)$ follows by inserting (1) and (2) in $w \bar{F}\left(\xi^{(w)}\right)=(1-w) \bar{G}\left(\xi^{(w)}\right)$. Also, $\mathrm{P}_{\mathrm{tx}}^{(w)} \in\left[\mathrm{P}_{\mathrm{tx}}^{(\mathrm{o})}, \mathrm{P}_{\mathrm{tx}}^{(\max )}\right]$ by virtue of the continuity of the Pareto front (Lemma 6 ).

Remark 7: From (8), the following remarks can be made. 1) $\mathrm{P}_{\mathrm{tx}}^{(w)}$ is given in closed-form. This is not possible, in general, from $\mathcal{P}_{\mathrm{T}}$ in (6). 2) $\mathrm{P}_{\mathrm{tx}}^{(w)}$ monotonically decreases in $\lambda_{\mathrm{BS}}$. This is because $\lambda_{\mathrm{BS}} \mathcal{L}\left(\lambda_{\mathrm{MT}} / \lambda_{\mathrm{BS}}\right)$ increases in $\lambda_{\mathrm{BS}}$ and $-\mathcal{T}$ decreases in $\lambda_{\mathrm{BS}}$. 3) The functional relation between $\mathrm{P}_{\mathrm{tx}}^{(w)}$ and $\lambda_{\mathrm{BS}}$ is fundamentally different compared with the case study where the coverage probability is maximized. In, e.g., the highly-loaded regime, i.e., $\mathcal{L}\left(\lambda_{\mathrm{MT}} / \lambda_{\mathrm{BS}}\right) \approx 1$, (8) yields 
$\mathrm{P}_{\mathrm{tx}}^{(w)} \propto 1 / \lambda_{\mathrm{BS}}$. If the coverage probability is maximized, we have $\mathrm{P}_{\mathrm{tx}} \propto \lambda_{\mathrm{BS}}^{-\beta / 2}$, where $\beta$ is the path-loss slope [8]. Notably, $\mathrm{P}_{\mathrm{tx}}^{(w)}$ in (8) is independent of the path-loss parameters.

Theorem 1: As a function of $\mathrm{P}_{\mathrm{tx}}$, the SE-EE Pareto front can be obtained from (2) by setting $\mathrm{P}_{\mathrm{tx}}$ as follows:

$$
\mathrm{P}_{\mathrm{tx}}=\left(\frac{\eta^{2 / \beta}}{\overline{\mathcal{S}}\left(\lambda_{\mathrm{BS}}\right)}\left[-\ln \left(1-\frac{\mathcal{S}\left(\lambda_{\mathrm{MT}} / \lambda_{\mathrm{BS}}\right) \mathrm{SE}}{\overline{\mathcal{L}}\left(\lambda_{\mathrm{BS}}\right)}\right)\right]\right)^{\beta / 2}
$$

where $\mathcal{S}\left(\lambda_{\mathrm{MT}} / \lambda_{\mathrm{BS}}\right)=1+\Upsilon \mathcal{L}\left(\lambda_{\mathrm{MT}} / \lambda_{\mathrm{BS}}\right), \overline{\mathcal{S}}\left(\lambda_{\mathrm{BS}}\right)=$ $\pi \lambda_{\mathrm{BS}} \mathcal{S}\left(\lambda_{\mathrm{MT}} / \lambda_{\mathrm{BS}}\right), \overline{\mathcal{L}}\left(\lambda_{\mathrm{BS}}\right)=\mathrm{B}_{\mathrm{W}} \rho_{\mathrm{D}} \lambda_{\mathrm{BS}} \mathcal{L}\left(\lambda_{\mathrm{MT}} / \lambda_{\mathrm{BS}}\right)$, and SE lies in the range $\mathrm{SE} \in\left[\mathrm{SE}\left(\xi^{(\mathrm{o})}\right), \mathrm{SE}_{\mathrm{m}}\right]$.

Proof: It follows by inserting $\mathrm{P}_{\mathrm{tx}}^{(w)}$ in (8) into (1) and (2), and by expressing $w$ as a function of the SE from (1). The range of values $\mathrm{SE} \in\left[\mathrm{SE}\left(\xi^{(\mathrm{o})}\right), \mathrm{SE}_{\mathrm{m}}\right]$ follows by virtue of the continuity of the Pareto front (as stated in Lemma 6).

In conclusion, the SE-EE Pareto front is obtained by inserting (9) into (2) and by plotting the curve for $\mathrm{SE} \in$ $\left[\mathrm{SE}\left(\xi^{(\mathrm{o})}\right), \mathrm{SE}_{\mathrm{m}}\right]$, which is decreasing in $\mathrm{SE}$ (Lemma 6).

\section{B. Case Study $\xi=\lambda_{\mathrm{BS}}$}

Lemma 9: Let $\xi=\lambda_{\mathrm{BS}}$ and $w \in\left[w_{l}, w_{u}\right]$, where $w_{l}$ and $w_{u}$ are defined in Lemma 7. Define $\lambda_{\mathrm{BS}}^{(\mathrm{o})}=\xi^{(o)}$. The unique solution, $\xi^{(w)}=\lambda_{\mathrm{BS}}^{(w)}$, of $\mathcal{P}_{\mathrm{ST}}$ in (7) is $\lambda_{\mathrm{BS}}^{(w)}=\lambda_{\mathrm{BS}}^{(\mathrm{o})}$ if $w=w_{u}$ and $\lambda_{\mathrm{BS}}^{(w)}=\lambda_{\mathrm{BS}}^{(\max )}$ if $w=w_{l}$. If $w \in\left(w_{l}, w_{u}\right), \lambda_{\mathrm{BS}}^{(w)} \in$ $\left(\lambda_{\mathrm{BS}}^{(\mathrm{o})}, \lambda_{\mathrm{BS}}^{(\max )}\right)$ is the unique solution of the equation:

$$
\mathrm{P}_{\text {grid }}=\mathrm{P}_{\text {grid }}\left(\lambda_{\mathrm{BS}}^{(w)}\right)=(1 / w-1)\left(\mathrm{SE}_{\mathrm{m}} / \mathrm{EE}_{\mathrm{o}}\right)
$$

where $\mathrm{P}_{\text {grid }}=\mathrm{P}_{\text {grid }}\left(\lambda_{\mathrm{BS}}^{(w)}\right)$ is defined in Table I.

Proof: The proof is similar to Lemma 8. Also, $\lambda_{\mathrm{BS}}^{(w)} \in$ $\left[\lambda_{\mathrm{BS}}^{(\mathrm{o})}, \lambda_{\mathrm{BS}}^{(\max )}\right]$ by virtue of continuity stated in Lemma 6.

By direct inspection of (10), similar comments as in Remark 7 can be made. They are not reported for brevity. As opposed to $\mathrm{P}_{\mathrm{tx}}^{(w)}$ in Lemma $8, \lambda_{\mathrm{BS}}^{(w)}$ cannot be formulated, in general, in closed-form. Two exceptions are studied as follows.

Corollary 1: If $\mathcal{L}\left(\lambda_{\mathrm{MT}} / \lambda_{\mathrm{BS}}\right) \approx 1$ (highly-loaded regime $), \lambda_{\mathrm{BS}}^{(w)}=\left((1 / w-1)\left(\mathrm{SE}_{\mathrm{m}} / \mathrm{EE}_{\mathrm{o}}\right)-\lambda_{\mathrm{MT}} \mathrm{P}_{\mathrm{c}}\right) / \mathrm{P}_{\mathrm{tx}}$. If $\mathcal{L}\left(\lambda_{\mathrm{MT}} / \lambda_{\mathrm{BS}}\right) \approx \lambda_{\mathrm{MT}} / \lambda_{\mathrm{BS}}$ (lightly-loaded regime), $\lambda_{\mathrm{BS}}^{(w)}=$ $\left((1 / w-1)\left(\mathrm{SE}_{\mathrm{m}} / \mathrm{EE}_{\mathrm{o}}\right)-\lambda_{\mathrm{MT}}\left(\mathrm{P}_{\mathrm{tx}}+\mathrm{P}_{\mathrm{c}}-\mathrm{P}_{\mathrm{i}}\right)\right) / \mathrm{P}_{\mathrm{i}}$.

Proof: It follows from Lemma 9 by solving (10).

Theorem 2: As a function of $\lambda_{\mathrm{BS}}$, the SE-EE Pareto front is constituted by the pairs $\left(\mathrm{SE}\left(\lambda_{\mathrm{BS}}^{(w)}\right), \mathrm{EE}\left(\lambda_{\mathrm{BS}}^{(w)}\right)\right)$ for $\lambda_{\mathrm{BS}}^{(w)} \in$ $\left[\lambda_{\mathrm{BS}}^{(\mathrm{o})}, \lambda_{\mathrm{BS}}^{(\max )}\right]$, where the SE and EE are given in (1) and (2).

Proof: It directly follows from Lemma 9.

It is worth noting that, even though $\lambda_{\mathrm{BS}}^{(w)}$ is, in general, not explicitly available, there is no need to compute it numerically. This is because, from Lemma 9 and by the continuity of the Pareto front, we have proved $\lambda_{\mathrm{BS}}^{(w)} \in\left[\lambda_{\mathrm{BS}}^{(\mathrm{o})}, \lambda_{\mathrm{BS}}^{(\max )}\right]$. As opposed to Theorem 1, it is not possible to express the EE as a function of the SE, because it is difficult to write $\lambda_{\mathrm{BS}}$ as a function of the SE from (1). Nevertheless, the SE-EE Pareto front is formulated without using any numerical methods.

\section{Numerical RESUlts}

The findings in Theorems 1, 2 are validated in Fig. 1. Setup: $\lambda_{\mathrm{MT}}=121 \cdot 10^{-6} \mathrm{MTs} / \mathrm{m}^{2}, \gamma_{\mathrm{D}}=\gamma_{\mathrm{A}}=5 \mathrm{~dB}, \mathrm{~B}_{\mathrm{W}}=20 \mathrm{MHz}$,
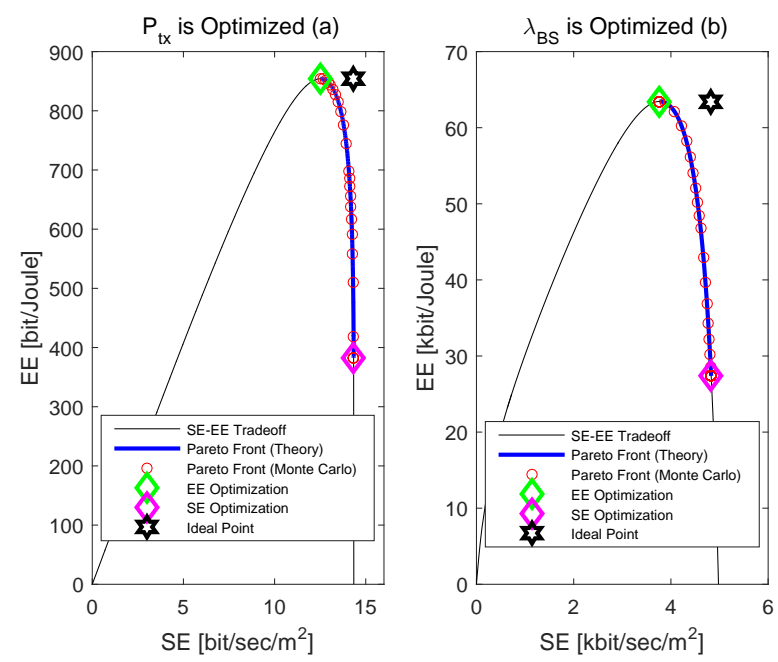

Fig. 1. SE-EE Pareto front: Monte Carlo simulations vs. theory.

$\beta=3.5, \mathrm{P}_{\mathrm{c}}=20 \mathrm{dBm}, \mathrm{P}_{\mathrm{i}}=10 \mathrm{dBm}, \kappa$ and $\mathrm{N}_{0}$ are set as in [8]. If $\xi=\mathrm{P}_{\mathrm{tx}}$, we set $\lambda_{\mathrm{BS}}=\left(\pi 500^{2}\right)^{-1} \mathrm{BSs} / \mathrm{m}^{2}, \mathrm{P}_{\mathrm{tx}}^{(\mathrm{min})}=0$ $\mathrm{dBm}, \mathrm{P}_{\mathrm{tx}}^{(\max )}=43 \mathrm{dBm}$. If $\xi=\lambda_{\mathrm{BS}}$, we set $\mathrm{P}_{\mathrm{tx}}=25 \mathrm{dBm}$, $\lambda_{\mathrm{BS}}^{(\min )}=\left(\pi 500^{2}\right)^{-1} \mathrm{BSs} / \mathrm{m}^{2}, \lambda_{\mathrm{BS}}^{(\max )}=\left(\pi 5^{2}\right)^{-1} \mathrm{BSs} / \mathrm{m}^{2}$.

The curve "SE-EE tradeoff" follows from (1), (2) for $\mathrm{P}_{\mathrm{tx}} \in$ $\left[\mathrm{P}_{\mathrm{tx}}^{(\min )}, \mathrm{P}_{\mathrm{tx}}^{(\max )}\right]$ in Fig. 1(a) and $\lambda_{\mathrm{BS}} \in\left[\lambda_{\mathrm{BS}}^{(\min )}, \lambda_{\mathrm{BS}}^{(\max )}\right]$ in Fig. 1(b). The curve "Pareto front (theory)" is computed from Theorems 1, 2. The markers "Pareto front (Monte Carlo)" are obtained by solving $\mathcal{P}_{\mathrm{T}}$ in (6) via exhaustive search. The other markers show the left and right extreme values of the Pareto front (Remark 6) and the "ideal point" $\left(\mathrm{SE}_{\mathrm{m}}, \mathrm{EE}_{\mathrm{o}}\right)$ [2].

Fig. 1 confirms the findings of Theorems 1, 2: The SE-EE Pareto front is constituted by a subset of points of the SE-EE trade-off curve, i.e., $\xi^{(w)} \in\left[\xi^{(\mathrm{o})}, \xi^{(\max )}\right] \subseteq\left[\xi^{(\min )}, \xi^{(\max )}\right]$.

\section{CONCLUSION}

In this letter, we have proved that the SE-EE Pareto front is constituted by a subset of points of the SE-EE trade-off curve, and that the functional dependency between the Pareto-optimal values of $\mathrm{P}_{\mathrm{tx}}$ and $\lambda_{\mathrm{BS}}$ is not the same as if the coverage is optimized. Also, the Pareto-optimal solutions either are given in closed-form or are the solution of a simple equation.

\section{REFERENCES}

[1] A. Zappone and E. Jorswieck, "Energy efficiency in wireless networks via fractional programming theory", Now Pub., vol. 11, Jun. 2015.

[2] K. Miettinen, Nonlinear Multiobjective Optimization, Kluver Pub., 1999.

[3] D. Tsilimantos et al., "Spectral and energy efficiency trade-offs in cellular networks", IEEE Trans. Wireless Commun., vol. 15, Jan. 2016.

[4] G. Zhao et al., "Joint energy-spectral-efficiency optimization of CoMP and BS deployment in dense large-scale cellular networks", IEEE Trans. Wireless Commun., vol. 16, no. 7, pp. 4832-4847, Jul. 2017.

[5] A. M. Alam et al., "Asymptotic analysis of area spectral efficiency and energy efficiency in PPP networks with SLNR precoder", IEEE Trans. Commun., vol. 65, no. 7, pp. 3172-3185, Jul. 2017.

[6] O. Aydin et al., "Energy-spectral efficiency tradeoffs in 5G multioperator networks with heterogeneous constraints", IEEE Trans. Wire less Commun., vol. 16, no. 9, pp. 5869-5881, Sep. 2017.

[7] Y. Hao et al., "On the energy and spectral efficiency tradeoff in massive MIMO-enabled HetNets with capacity-constrained backhaul links", IEEE Trans. Commun., vol. 65, pp. 4720-4733, Nov. 2017.

[8] M. Di Renzo et al., "System-level modeling \& optimization of the energy efficiency in cellular networks - A stochastic geometry framework", IEEE Trans. Wireless Commun., vol. 17, pp. 2539-2556, Apr. 2018.

[9] A Cambini et al., Generalized Convexity \& Optimization, Springer, 2009.

[10] A. Daniilidis et al., "Connectedness of the efficient set for three-objective quasiconcave naximization problems", J. Opt. Th. \& Appl., vol. 93, 1997. 